\title{
Intermédialités
}

Histoire et théorie des arts, des lettres et des techniques

Intermediality

History and Theory of the Arts, Literature and Technologies

\section{Aimer, s’aimer à s'y perdre? Les jeux spéculaires de Cahun-Moore}

\section{Andrea Oberhuber}

Numéro 4, automne 2004

\section{Aimer}

Loving

URI : https://id.erudit.org/iderudit/1005478ar

DOI : https://doi.org/10.7202/1005478ar

Aller au sommaire du numéro

\section{Éditeur(s)}

Centre de recherche sur l'intermédialité

ISSN

1705-8546 (imprimé)

1920-3136 (numérique)

Découvrir la revue

Citer cet article

Oberhuber, A. (2004). Aimer, s'aimer à s'y perdre? Les jeux spéculaires de Cahun-Moore. Intermédialités / Intermediality, (4), 87-114.

https://doi.org/10.7202/1005478ar
Résumé de l'article

Que penser d'une jeune artiste qui se présente tantôt en Méduse ou en beaute orientale, tantôt en bouddha, en haltérophile ou en Gretchen? Que penser de cet autoportrait dédoublé " en damier " qui fait écho au portrait d'une femme " en rayures ", celui-ci également dédoublé? Comment décoder des photomontages - tous plus énigmatiques les uns que les autres - conçus en collaboration avec cette même femme " en rayures ", et qui se retrouvent intercalés dans un texte intitulé Aveux non avenus? Que signifie " aimer », lorsque l'être aimé est notre alter ego? Cette histoire d'amour entre soi et la projection de soi peut-elle éviter l'abîme? Cet article propose de réfléchir sur la notion d'" aimer " chez Claude Cahun et Suzanne Malherbe alias Marcel Moore, en interrogeant le côté " narcissique » et autoréflexif que révèlent la plupart des autoportraits, l'autobiographie et les photomontages, d'une part, et le désir lesbien stigmatisé à l'époque comme un "faux masque », d'autre part. Dans un deuxième temps, il s'intéressera à ce couple symbiotique que forment l'auteure-photographe Cahun et la graphiste-peintre Moore, symbiose artistique qui leur permet de créer des oeuvres à leur image. 


\title{
Aimer, s'aimer à s'y perdre? Les jeux spéculaires de Cahun-Moore
}

\author{
Andrea Oberhuber
}

\begin{abstract}
tonnant paradoxe que celui de Claude Cahun - auteure, photographe, actrice de théâtre et essayiste - qui, après avoir été présente sur la scène culturelle de son époque, se retrouve après sa mort parmi les «oubliés» de l'historiographie littéraire et artistique ${ }^{1}$. Il a fallu attendre les années 1980, le renouveau de l'intérêt pour le surréalisme, notamment pour le rôle des femmes dans les avant-gardes de l'entre-deux-guerres, attendre surtout les débats autour du gender pour voir ressusciter Claude Cahun, née Lucy Schwob en 1894, fille de l'éditeur Maurice Schwob et nièce du poète symboliste Marcel Schwob. Ce n'est toutefois pas un hasard si Cahun a été redécouverte il y a une vingtaine d'années dans le cadre des gender studies nord-américaines pour, instantanément, en devenir l'un des enfants chéris. Surtout pour les historiennes de l'art

1. Les raisons de sa marginalisation passée et de son succès présent sont multiples: François Leperlier, biographe et «redécouvreur» de Claude Cahun, en trouve l'explication dans son "propos de troubler les indices, de se soustraire aux identifications sommaires» et dans son jeu avec la "plus intime étrangeté » (François Leperlier, Claude Cahun: l'écart et la métamorphose, Paris, Jean-Michel Place, 1992, p. 13); Laura Cottingham explique l'oubli de Cahun par son désir lesbien («Betrachtungen zu Claude Cahun», dans Claude Cahun. Bilder, catalogue d'exposition, Heike Ander et Dirk Snauwaert (dir.), Munich, Schirmer \& Mosel, 1997, p. XX-XXII) et Carolyn Dean, par sa non-participation aux spectaculaires actions des surréalistes ( CClaude Cahun's Double », Yale French Studies, $n^{\circ}$ 90, décembre 1996, p. 71-72). La conjonction de toutes ces positions dérangeantes, difficiles à classifier, a certainement contribué à reléguer cette artiste hors du commun aux oubliettes de l'histoire.
\end{abstract}


qui s'intéressent aux aspects de l'autoreprésentation et de la performance en photographie, aux rapports entre féminisme et postmodernisme ${ }^{2}$, Cahun est une figure clé du modernisme français tel qu'il s'est manifesté entre 1900 et 1940, tantôt au sein des mouvements avant-gardistes, tantôt en leurs marges.

Si Cahun est donc sortie des tiroirs de l'oubli auquel l'histoire culturelle l'avait reléguée, il n'en est pas de même pour sa compagne de vie, la peintre et graphiste Suzanne Malherbe. C'est pourtant avec celle qui signait ses œuvres Marcel Moore (ou Moore tout court) que Cahun formait l'un de ces couples symbiotiques qui animaient le milieu intellectuel et artistique du «Paris Lesbos », ces célèbres salons de femmes installés sur la rive gauche, loin des grands salons aristocratiques et grand-bourgeois du Faubourg-Saint-Germain³. Qui imaginerait Gertrude Stein sans son alter ego Alice Toklas (immortalisée dans la célèbre Autobiography of Alice B. Toklas ${ }^{4}$ ), la poétesse Renée Vivien sans l'amazone par excellence, Natalie Clifford, ou encore Colette sans Missy à ses côtés, après son divorce de Willy, sur les scènes du music-hall parisien? Dans le cas de Claude Cahun et de Marcel Moore, le double pseudonyme aurait-il joué en la défaveur de ces deux artistes pour qui le «shifting gender» tel qu'il se manifeste dans les écrits, les autoportraits et les photomontages devenait la traduction parfaite d'un «shifting $I^{5}$ »? La destruction en 1944 d'une bonne partie de leur

2. En témoignent, à titre d'exemples, les ouvrages suivants: Renée Riese Hubert, Magnifying Mirrors. Women, Surrealism, \& Partnership, Lincoln, University of Nebraska Press, 1994; Amelia Jones, Postmodernism and the En-Gendering of Marcel Duchamp, Cambridge, Cambridge University Press, 1994; Whitney Chadwick (dir.), Mirror Images: Women, Surrealism, and Self-representation, Cambridge, London, MIT Press, 1998; le catalogue d'exposition Rrose is a Rrose is a Rrose. Gender Performance in Photography, Jennifer Blessing (dir.), New York, Guggenheim Museum Publications, 1997; Rosalind Krauss, Bachelors, Cambridge, MIT Press, coll. «October», 1999; Shelley Rice, Inverted Odysseys: Claude Cahun, Maya Deren, Cindy Sherman, Cambridge, MIT Press, 2000; Jennifer Mundy, Surrealism. Desire Unbound, Princeton, Princeton University Press, 2001.

3. Pour ce qui concerne les différences entre les salons animés par les «Américaines » (pour la plupart autoexilées à Paris) et ceux tenus par la vieille aristocratie française ou la grande-bourgeoisie, voir Shari Benstock, Femmes de la rive gauche: Paris 1900-1940, trad. Jacqueline Carnaud, Paris, Éditions des femmes, coll. «Essai», 1987, p. 50-107.

4. Gertrude Stein, Alice B. Toklas, The Autobiography of Alice B. Toklas, New York, Literary Guild, 1933.

5. Honor Lasalle et Abigail Solomon-Godeau, «Surrealist Confession: Claude Cahun's Photomontages ", Afterimage, vol. 19, nº 8, mars 1992, p. 10. 
œuvre par des soldats national-socialistes, lors du pillage de leur résidence d'exil sur l'île de Jersey, aurait-elle été à l'origine d'une memoria défaillante jusqu'à tout récemment en ce qui concerne la transmission de l'œuvre littéraire et photographique de Cahun-Moore ${ }^{6}$ ? Ou serait-ce le simple fait que le discours culturel, dans sa conception de l'artiste comme individu créateur, n'admette que difficilement la création collective ${ }^{7}$, pratiquée par Cahun et Moore en tant que couple symbiotique?

Cette réception partielle - Cahun sans Moore - paraît d'autant plus fâcheuse que le couple transgressait sans cesse les limites du soi, afin d'explorer les multiples facettes du Même et ce, jusque dans les abîmes de l'altérité. Je pose l'hypothèse qu'une grande partie de l'œuvre de Cahun est, en réalité, inconcevable sans le concours de Moore. Marqué d'incessants jeux spéculaires, leur travail de création est indissociable de leur histoire d'amour fusionnel: Cahun, la cadette, comme double de Moore, ou l'inverse ${ }^{8}$. Dès la première œuvre littéraire et jusqu'aux derniers autoportraits, le lecteur-spectateur est confronté à la polymorphie d'un «je» en métamorphose permanente, à une transformation qui s'effectue sous le regard bienveillant - et, probablement, contrôlant d'un point de vue esthétique - de sa compagne. Ensemble, les deux

6. À la suite de leur arrestation par la Gestapo, on croyait Cahun et Moore déportées et finalement mortes dans un camp de concentration. Pour les méandres de l'oubli et de la redécouverte de cette œuvre, voir plus en détail Thérèse Lichtenstein, «A Mutable Mirror: Claude Cahun », Artforum, vol. 30, nº 8, avril 1992, p. 65.

7. Les études dans ce domaine, plus particulièrement en ce qui concerne la collaboration entre femmes, ne sont pas légion. Citons à titre d'exemples le travail de MarieJo Bonnet, Essai sur le couple de femmes dans l'art, Paris, Éditions Blanche, 2000, et, sur les couples artistes dans les mouvements dadaïstes et surréalistes, l'ouvrage de Renée Riese Hubert, Magnifying Mirrors. Women, Surrealism, \& Partnership.

8. Dans Aveux non avenus, par exemple, la figure du double, sans manquer d'ambiguiité, est associée au motif de la sœur jumelle: «tu ne pouvais exister sans ta fausse jumelle. Vous avez partie liée. Tu ne peux l'exterminer sans t’abolir» (Claude Cahun, Aveux non avenus, Paris, Éditions du Carrefour, 1930, p. 14), à celui de l'hermaphrodite et du siamois: «Les amants trop heureux forment un couple pareil au monstre hermaphrodite ou encore aux frères siamois (p. 33), ou encore à celui des cheveux emmêlés qu'il faut couper le lendemain matin afin de les dissocier : "Nos cheveux se sont emmêlés tant et tant cette nuit, qu'au matin - pour en finir - nous avons dû nous faire tondre.» (p. 145) Désormais les références à cet ouvrage seront indiquées par le sigle «ANA », suivi de la page et placées entre parenthèses dans le corps du texte. 
artistes sondent les corrélations entre les mots et les images, le corps, le sexe et leurs représentations sociales, le sujet et l'identité, la mise en théâtre de soi et le désir lesbien, s'inscrivant ainsi dans les thèmes hautement en vogue dans les milieux de la rive gauche ${ }^{9}$ et qui, sous l'enseigne du postmodernisme, domineront le discours sur les catégories du sexe et du gender ${ }^{10}$. Il s'agira ici de montrer l'enjeu du couple amoureux pour la création d'une œuvre protéiforme qui souscrit aux prémisses littéraires et picturales des avant-gardes de l'entre-deuxguerres tout en gardant une certaine distance, un regard oblique sur la scène artistique dominante. Que signifie «aimer» et «s'autoreprésenter» lorsque l'être aimé est notre alter ego? Cette histoire d'amour entre soi et la projection de soi sous forme de textes littéraires, d'autoportraits, de photomontages et d'objets surréalistes peut-elle éviter l'abîme? Et que signifie «aimer une femme » dans une société qui valorise le discours masculin, la double morale et le modèle hétérosexuel?

9. Le Paris de l'entre-deux-guerres est un lieu effervescent pour toute une génération de femmes dont un grand nombre vient de l'autre côté de l'Atlantique et que l'on désignera comme «Amazones» ou «Américaines». À propos des intellectuelles américaines et anglaises expatriées à Paris entre 1900 et 1940 (Edith Wharton, Djuna Barnes, Natalie Clifford, Janet Flanner, Sylvia Beach et Gertrude Stein, entre autres), voir Shari Benstock, Femmes de la rive gauche: Paris 1900-1940; Andrea Weiss, Paris was a Woman, San Francisco, London, Harper Collins Publishers, 1995. Dès 1922, Claude Cahun et Marcel Moore animaient leur propre salon à Montparnasse que fréquentait l'intelligentsia de l'époque, de Georges Bataille à Sylvia Beach, de Lise Deharme à Tristan Tzara en passant par André Breton, Robert Desnos, Jacques Lacan, Henri Michaux et Jacques Viot. C'est là surtout que Cahun et Moore vivaient leur altérité et leur goût pour le travestissement, mais également à la librairie d'Adrienne Monnier où le couple côtoyait Sylvia Beach, Jane Heap, Margaret Anderson, tout comme il devait y rencontrer à l'occasion Aragon, Benjamin, Claudel, Jacob et Soupault. Soulignons que, à l'extérieur de ces lieux «protégés », la tolérance à l'égard des couples lesbiens était plutôt restreinte. N'y faisait pas exception le cercle des surréalistes homophobes - autour de Breton et d'Aragon - qui voyaient d'un mauvais œil l'apparition du couple extravagant CahunMoore au Cyrano, haut lieu du mouvement surréaliste durant les années parisiennes. Pour le cercle amical du couple et ses échanges littéraires et artistiques, voir François Leperlier, Claude Cahun : l'écart et la métamorphose, Paris, Éditions Jean-Michel Place, 1992 , p. 105 .

10. Est-il nécessaire de rappeler le rôle de précurseur qu’a joué Marcel Duchamp alias Rrose Sélavy dans la remise en question de la construction sociale des rôles sexués, des attributs «féminin »/«masculin», de la mascarade comme stratégie subversive et de 


\section{«L'ACTE MÊME EST GUVRE DE CHAIR"" ": AMOUR ET DÉSIR LESBIEN DANS L'ENTRE-DEUX-GUERRES}

Après le bimorphisme caractéristique de la différence sexuelle du XIX ${ }^{\mathrm{e}}$ siècle, l'apparence féminine évolue, à partir du tournant du siècle, vers une silhouette de plus en plus androgyne à la Belle Époque. La peur de la virilisation des femmes est une hantise que les psychiatres partageront avec une grande partie de la société, dont également les féministes «réformistes». Rares sont cependant les femmes dans la vie de tous les jours, contrairement au riche imaginaire fantasmatique des peintres et des écrivains, qui osent afficher ouvertement, à l'encontre de leur sexe féminin, une image «masculine». Madeleine Pelletier, femme médecin et figure de proue du féminisme combattant de l'époque, est l'exception la plus flagrante ${ }^{12}$; pour elle, la virilisation, en passant par le costume et le chapeau masculin, est le gage de l'émancipation de la femme. Les autres femmes «libérées » - un petit pourcentage de la population seulement, issu notamment des milieux bourgeois - évoluent vers l'allure androgyne de la garçonne, mythe des années folles ${ }^{13}$. Mais l'époque n'est pas à un paradoxe près. Les divers mouvements féministes demeurent longtemps encore prisonniers d'une «idéologie qui façonne des images de femmes acceptables, reconnues socialement par leur lien à l'Autre masculin, le mari et ses enfants ${ }^{14}{ }$. D'un autre côté, dès 1912, l'archétype de l'amazone ${ }^{15}$ investit l'imaginaire social de la

l'artiste comme «mère »/«père » d'une œuvre d'art? Voir plus en détail le chapitre «The Ambivalence of Rrose Sélavy and the (Male) Artist as "Only the Mother of the Work" » que consacre à cette problématique Amelia Jones, Postmodernism and the En-Gendering of Marcel Duchamp, p. 146-190.

11. ANA, p. 118 .

12. L'historienne Christine Bard a dirigé un ouvrage collectif consacré à l'une des féministes les plus radicales de l'entre-deux-guerres: Christine Bard (dir.), Madeleine Pelletier (1874-1939). Logique et infortunes d'un combat pour l'égalité, actes du colloque de Paris (5-6 décembre 1991), Paris, Éditions Côté-femmes, coll. «Des femmes dans l'histoire ", 1992.

13. Voir l'étude de cette figure mythique, richement illustrée d'un point de vue iconographique, par Christine Bard, Les garçonnes: modes et fantasmes des années folles, Paris, Éditions Flammarion, coll. "Génération », 1998.

14. Marie-Jo Bonnet, Les relations amoureuses entre les femmes: $\mathrm{XVI}^{e}-\mathrm{XX}{ }^{e}$ siècle, Paris, Éditions Odile Jacob, 2001 [1995], p. 325.

15. Modigliani est l'un des premiers à avoir immortalisé une Amazone en 1909. Chana Orloff, en 1916, donna le même titre à une sculpture montrant une Amazone sur son cheval. 
«femme» en la personne de Natalie Clifford, auteure de Pensées d'une amazone $^{16}$ et animatrice de l'un des plus célèbres salons parisiens. C'est le caractère iconoclaste et contestataire de ce nouveau modèle féminin qui va à l'encontre des représentations attendues.

À l'image de la New woman engendrée par la Première Guerre mondiale, ces «amazones » rejoindront naturellement les courants avant-gardistes porteurs de toutes les idées neuves, notamment en littérature et dans les arts. Si elles sont nombreuses à participer aux mouvements d'avant-garde, c'est qu'elles y trouvent le moyen d'expression d'un nouveau regard sur le monde, d'un nouveau mode de vie débarrassé de certaines contraintes sociales. Il suffit de penser aux travaux de la cinéaste Germaine Dulac, de la danseuse Loïe Fuller, de la peintre Valentine Penrose, de la chanteuse Suzy Solidor, des auteures et journalistes Georgette Leblanc, Margaret Anderson et Jane Heap pour mesurer l'apport des femmes dans les avant-gardes de l'entre-deux-guerres, cette part que Lea Vergine qualifie d' «autre moitié de l'avant-garde» et Jennifer E. Milligan, de «forgotten generation ${ }^{17}$ ». Durant cette période fertile en échange interartistique et favorable à l'expérience pluridisciplinaire, les artistes, peintres et écrivaines affrontent le tabou de la représentation de l'érotisme lesbien. En témoignent les œuvres de Chana Orloff, de Romaine Brooks, de Marie Laurencin et, bien sûr, du couple Cahun-Moore. C'est en 1925, lors de l'Exposition internationale des arts décoratifs et industriels modernes, que le saphisme nouveau style affiche publiquement ses couleurs : les visiteurs du Pavillon du livre sont accueillis par une statue de Bourdelle représentant la poétesse Sapho avec sa lyre comme symbole de la femme créatrice ${ }^{18}$.

16. Natalie Clifford Barney, Pensées d'une amazone, Paris, Émile-Paul frères, 1920.

17. Lea Vergine, L'autre moitié de l'avant-garde 1910-1940: femmes peintres et femmes sculpteurs dans les mouvements d'avant-garde historiques, trad. Mireille Zanuttini, Paris, Éditions Des femmes, 1982 [1980]; Jennifer E. Milligan, The Forgotten Generation: French Women Writers of the Inter-War Period, Oxford, New York, Berg Press, 1996.

18. Pour l'évolution du saphisme Belle Époque vers le lesbianisme de l'entre-deuxguerres, voir plus en détail Marie-Jo Bonnet, Les relations amoureuses entre les femmes, p. 321-334; on lira également avec intérêt le chapitre «Le charme de la décadence» que consacre Florence Tamagne à la corrélation entre dandy et homosexuel, entre femme fatale et lesbienne: Florence Tamagne, Mauvais genre? Une histoire des représentations de l'homosexualité, Paris, Éditions de La Martinière, 2001, coll. «Les reflets du savoir », p. 100-121. Le désir lesbien semble sortir du secret; il exerce surtout une véritable fascination sur les artistes hommes, si l'on pense au leitmotiv des «Deux amies» qui revient 
$\mathrm{Si}$, dans cette période de l'entre-deux-guerres, un plus grand nombre de femmes s'expriment à travers l'art et l'écriture, c'est sans doute aussi parce que d'autres terrains d'action - la voie politique, notamment - leurs sont interdits dans un pays qui ne leur accorde toujours pas le droit de vote en les excluant ainsi de l'«universel» masculin. Plusieurs d'entre elles réalisent leurs œuvres dans la «sphère privée » avant de connaître, plusieurs décennies après seulement, une reconnaissance publique ${ }^{19}$. Vivre, écrire et créer en marge de la scène dominante, se situer dans un «dedans » plutôt que dans le «dehors », contribue forcément à développer non seulement une vision autre du réel, mais également une esthétique différente.

\section{Cahun et Moore, une relation érotico-artistique}

La relation amoureuse entre Cahun et Moore et leur étroite collaboration s'inscrivent indubitablement dans le contexte de la création d'un nouvel imaginaire, au-delà des frontières normatives de l'image féminine traditionnelle. Leur amour n'est pas un secret, certes, mais il se fait plus discret que celui qui déchire par exemple Natalie Barney et Renée Vivien, plus égalitaire aussi que celui qui caractérise le couple Stein-Toklas ${ }^{20}$. Pour saisir les nuances de la

sans cesse chez Foujita, Pascin, Bourdelle, Chemiot ou Toulouse-Lautrec, et dont le registre va du morbide au sentimental en passant par le sensuel voyeuriste. Contrairement au désir lesbien, l'homosexualité masculine investit plutôt la littérature, d'Oscar Wilde à Marcel Proust ou André Gide.

19. Les rapports entre le centre et la périphérie sont particulièrement problématiques dans ces années de l'entre-deux-guerres. C'est ce que fait remarquer Susan Rubin Suleiman à propos de la double marginalité à laquelle sont reléguées les femmes dans les mouvements avant-gardistes: Susan Rubin Suleiman, Subversive Intent: Gender, Politics, and the Avant-Garde, Cambridge, Massachusetts, London, Harvard University Press, 1990, p. 11-32. La réévaluation du rôle des femmes dans le dadaïsme et le surréalisme se fait en effet seulement dans les années 1990. Voir Georgiana M. M. Colvile et Katherine Conley (dir.), La femme s'entête : la part du féminin dans le surréalisme, actes du Colloque de Cerisy-la-Salle (août 1997), Paris, Lachenal \& Ritter, coll. "Pleine marge», 1998; Georgiana M. M. Colvile, Scandaleusement d'elles: trente-quatre femmes surréalistes, Paris, Éditions Jean-Michel Place, 1999.

20. Cahun ne thématise quasiment jamais explicitement le thème de l'homosexualité ni du désir lesbien; il faut savoir le déchiffrer, le lire entre les lignes. Un seul texte essayistique - sa réponse à une enquête menée par la revue L'amitié en avril 1925, trois ans avant les célèbres entretiens des surréalistes sur l'h omosexualité publiés dans $L a$ 


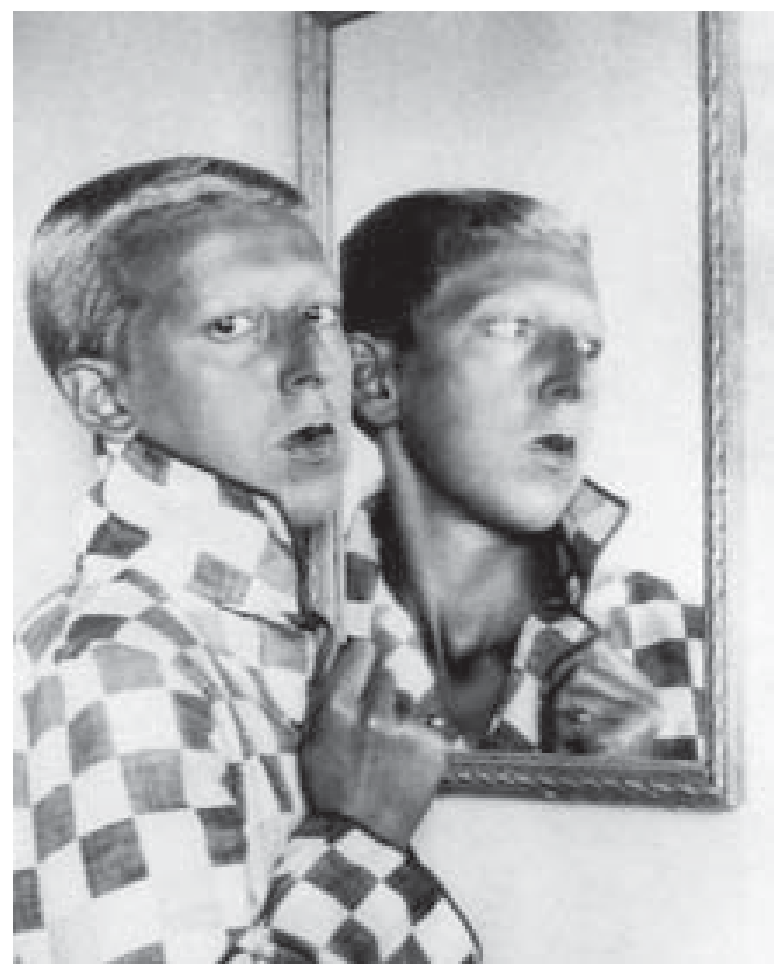

Fig. 1. Claude Cahun, Autoportrait, 1928, coll. Musée des Beaux-Arts, Nantes, coll. privée, Évreux.

complémentarité entre Cahun et Moore, il faut s'attarder sur les détails de leur autoreprésentation qui emprunte le plus souvent la voie de l'autoportrait photographique. L'emblématique «Autoportrait ${ }^{21}$ » (fig. 1) de Claude Cahun devant un miroir, aux cheveux courts et au regard tourné vers le spectateur, qui

révolution surréaliste - , ainsi que la nouvelle restée inédite "Salmacis la suffragette » renvoient à une orientation sexuelle et un mode de vie marquant clairement la différence. Pour plus de détails, voir Andrea Oberhuber, "Que Salmacis surtout évite Salmacis !" Claude Cahuns literarisch-fotografische Verkörperungen des Anderen », dans Dirk Naguschweski et Sabine Schrader (dir.), Sehen, Lesen, Begehren: Homosexualität in französischer Literatur und Kultur, Berlin, Edition Tranvía, Verlag Walter Frey, 2001, p. 67-81; Laura Cottingham, Cherchez Claude Cahun, Lyon, Éditions Carobella exnatura, 2002, p. 16-29.

21. Reproduite sur la page de couverture de plusieurs ouvrages consacrés aux femmes artistes dans le surréalisme, cet autoportrait est devenu l'icône du travail photogra- 


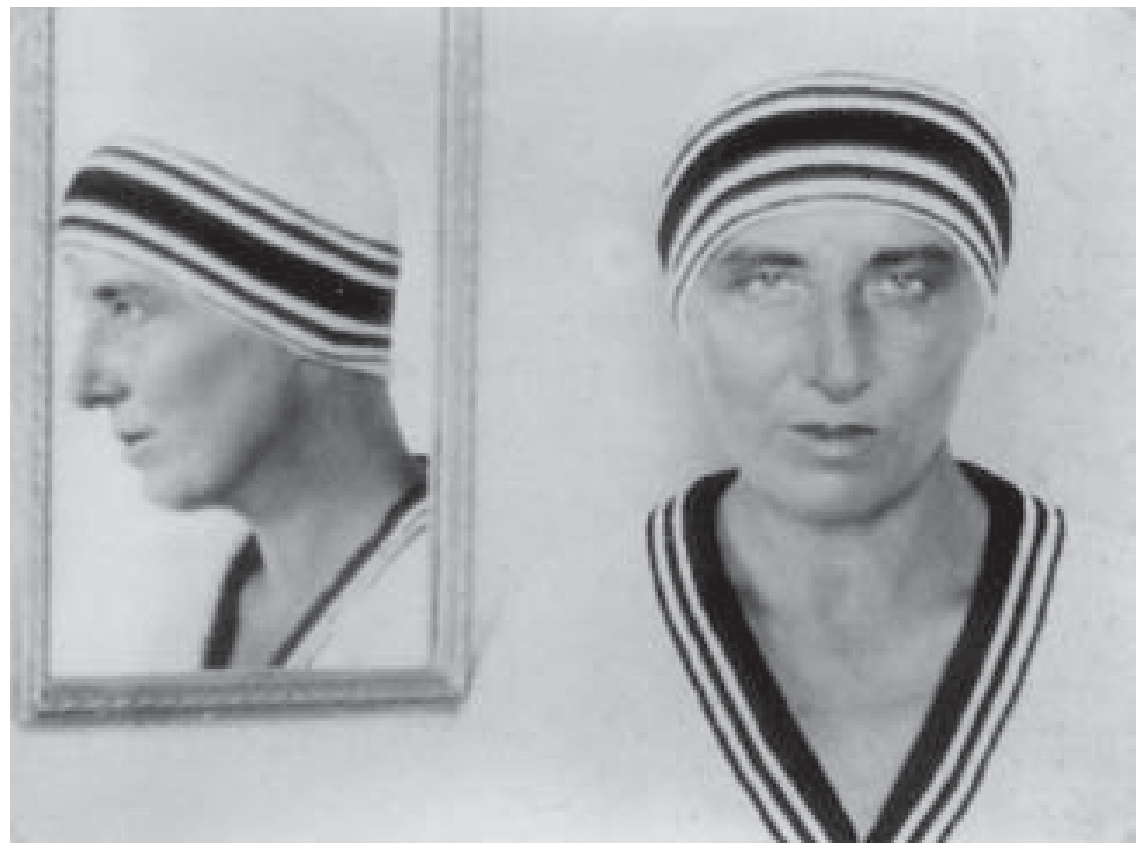

Fig. 2. Claude Cahun, Suzanne Malherbe, 1928, coll. privée, Paris.

la montre habillée d'une veste en motif à damier blanc et noir est, en vérité, indissociable de l'(auto)portrait «Suzanne Malherbe», réalisé par ailleurs la même année, sur lequel elle porte un pull-over et un bonnet blancs rehaussés tous deux par des rayures noires et blanches (fig. 2 2 $^{22}$. Loin de vouloir se ressembler comme des jumelles siamoises, loin donc aussi du mythe de Narcisse et de sa sœur - je reviendrai sur ce point crucial de l'interprétation des autoportraits et des photomontages ${ }^{23}$-, le moi et son alter ego affichent leur différence dans

phique de Cahun. Pour toutes les images auxquelles je fais ici référence, je renvoie, pour l'excellente qualité de reproduction des photographies, au catalogue d'exposition Claude Cahun. Bilder. L'autoportrait en question est reproduit à la p. 29. On pourra également se renseigner sur les autoportraits et les photomontages grâce au catalogue Claude Cahun photographe, édité par le Musée d'art moderne de la ville de Paris, Paris, Éditions Jean-Michel Place, 1995, qui présente un plus petit nombre d'images de qualité moindre.

22. Claude Cahun. Bilder, p. 105.

23. C'est avec le concours de Marcel Moore que Cahun perfectionne la technique du photomontage, notamment pour réaliser les héliogravures d’Aveux non avenus. Plus 
la complémentarité. Un second autoportrait appuiera encore mieux cette vision de deux moi distincts, indépendants, qui ne sont jamais tout à fait les mêmes ni tout à fait autres. Cette fois, Cahun et Moore se retrouvent réunies dans le même autoportrait intitulé «Le Croisic ${ }^{24}$ (fig. 3). Divisée en deux par la mise en scène du cadre devant lequel la photographie a été prise, l’image semble «consacrer» les deux femmes: elles sont debout, habillées respectivement en tailleur et en robe et «coiffées » de la même façon, s'appuyant légèrement sur l'estrade d'un balcon qui ouvre la vue sur la mer et l'horizon au large. Chacune d'elle occupe une moitié de l'image - Moore la moitié gauche, Cahun la moitié droite - comme les deux parties d'un même cerveau ou les deux chambres d'un même cœur. Cependant, notons-le tout de suite, ce type d'autoreprésentation est chose rarissime dans l'œuvre qui nous est restée. Ce qui témoigne, d'une autre façon et moins ponctuellement, de leur esprit commun, c'est le travail collectif qu'effectuent l'auteure et la graphiste-peintre pour Aveux non avenus $^{25}$, œuvre autobiographique, voire autofictionnelle avant la lettre, à l'intérieur de laquelle sont intercalés dix photomontages. La symbiose artistique au sein de laquelle les deux femmes donnent et reçoivent se révèle l'univers idéal leur permettant de créer des œuvres à leur image. Les photomontages-cryptogrammes, recyclage de certains autoportraits assemblés autrement, d'images diverses, de phrases isolées ou de bribes textuelles, sont réalisés entre 1929 et 1930 en étroite collaboration entre Cahun et Moore, ce qu'explique clairement le paratexte d'Aveux non avenus: «illustré d'héliogravures composées par Moore d'après les projets de l'auteur». Le «Tableau I» (ANA, s.p., fig. 4) immortalise par ailleurs, en bas à droite, la signature de Moore, deuxième trace visible donc incontournable - d'une démarche qui veut être perçue comme un travail bicéphale.

Que ce soit dans le cadre de l'illustration de la première œuvre littéraire de Claude Cahun, Vues et visions ${ }^{26}$, des nombreuses mises en scène de soi

tard, cette même technique se retrouvera exploitée dans la collaboration entre Cahun et Lise Deharme dans son livre pour enfants Le cœur de pic, Paris, Éditions José Corti, 1937 .

24. Claude Cahun. Bilder, p. 104.

25. Les œuvres littéraires de Cahun ont récemment fait l'objet d'une réédition: Claude Cahun, Écrits, François Leperlier (éd.), Paris, Éditions Jean-Michel Place, 2002.

26. Publiés dans un premier temps en 1914 dans le Mercure de France, ces poèmes en prose ont été regroupés cinq ans plus tard dans un recueil paru à Paris chez Georges Crès. 


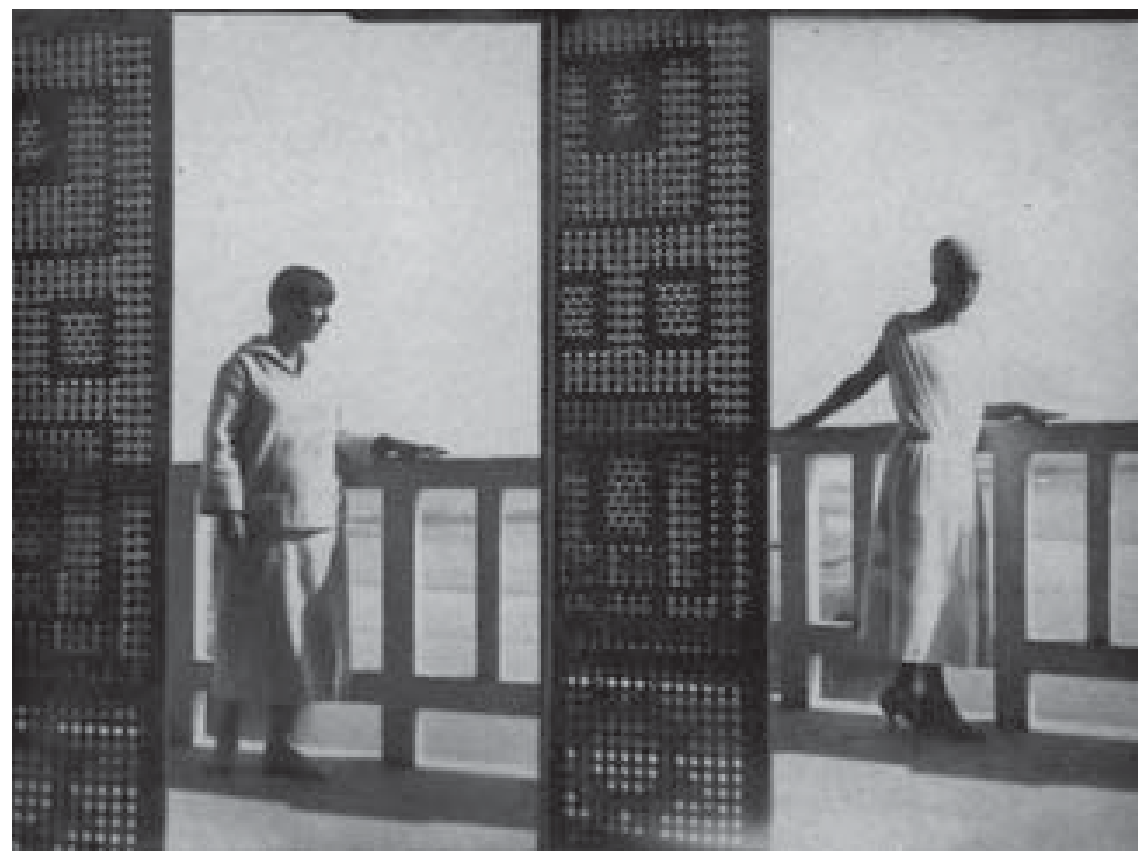

Fig. 3. Claude Cahun, Marcel Moore, Le Croisic, 1921, coll. privée, Paris.

devant la caméra ou des photomontages pour Aveux non avenus, la collaboration féminine sera le moyen de se soustraire au triple rôle de la muse-modèlemaîtresse auquel s'astreignirent plusieurs artistes surréalistes, du moins durant une période de leur carrière ${ }^{27}$. Nul doute cependant que ce moi scintillant et insaisissable des autoportraits se conçoit aussi comme un modèle. Mais il s'agit là d'un modèle qui, à travers de multiples visages, se construit sans cesse différemment en déconstruisant les attributs d'une féminité et d'une masculinité socialement codifiées. Le regard posé sur un autre modèle, transformé ainsi en

27. Voir à ce propos la vive critique que formule Xavière Gauthier dans sa lecture du groupe surréaliste et du traitement que Breton, Aragon et Éluard, pour ne citer que le noyau dur du groupe, réservent aux femmes du mouvement: Xavière Gauthier, Surréalisme et sexualité, Paris, Éditions Gallimard, coll. «Idées», 1971; voir également les chapitres «À la recherche de la muse» et «La femme, muse et artiste» que consacre Whitney Chadwick à l'image de la femme, quelque peu restrictive, propagée dans les textes littéraires et poétiques des mêmes surréalistes: Whitney Chadwick, Les femmes dans le mouvement surréaliste, Paris, Éditions du Chêne, 1986 [1985], p. 13-102. 
muse, se reflète dans l'œil de celle qui, hors-cadre, observe, dirige et mène à bien ce projet d'une métamorphose sans fin. Qui, dans ce jeu de rôles parfaitement théâtral, est l'artiste créateur, qui est la muse inspiratrice? Force est de constater que les deux déserteurs d'une répartition stéréotypée des rôles de Pygmalion et de Galatée imaginent un nouveau concept d'art et de vie au-delà des frontières traditionnelles, là où, justement, l'art se mêle à la vie et la vie à l'art. Cette esthétique transgressive au sens premier du terme n'était-elle pas au cœur du grand rêve des avant-gardes dadaïste et surréaliste? Ne s'agissait-il pas avant tout d'abattre les cloisons entre les disciplines et les genres, rendre leurs parois plus poreuses?

S'il convient de reprendre, afin de l'approfondir, l'idée de l'artiste et de son modèle, c'est en des termes de réciprocité. Car il est certain que le travail de la graphiste et peintre Moore, plus âgée et plus expérimentée dans le domaine de l'art, a inspiré l'écriture des premiers poèmes en prose de Cahun. Ainsi, la conception en diptyque de Vues et visions témoigne de l'influence picturale. Les poèmes sont nourris par le principe du double et du dédoublement, d'un prétendu original et de sa variation qui passe par le biais de la transposition spatiotemporelle. Accompagnés chacun d'un dessin servant de cadre, les vingtcinq poèmes en prose évoquent sur la page de gauche des observations, des impressions, des réflexions d'un moi qui, le vague à l'âme, passe quelques jours au bord de la mer, au Croisic; sur la page de droite, la même scène se voit transposée dans l'Antiquité gréco-latine. Alors que, dans la scène originale, le «je» semble être toujours le même, dans la variation, il prend d'autres noms, d'autres identités. Tels des enluminures, les dessins ${ }^{28}$ - concrets ou abstraits encadrent les deux scènes. Complémentaires, celles-ci se font face comme dans un jeu de miroir qui aurait la particularité de transformer, de varier, de substituer certains mots ou tournures de phrase. Mots et images reflétés, réalité filtrée à travers le regard et transformée par les moyens de l'écriture, voilà des caractéristiques d'une œuvre qui n'aura de cesse d'explorer plus avant ces effets de dédoublement. La propension au jeu verbal, le principe du détournement de sens, de même que l'idée du double annoncent une esthétique intermédiale 29

28. Certains de ces dessins décoratifs rappellent le style d'Aubrey Beardsley (qui affichait - rappelons-le - un net penchant pour les représentations saphiques), d'autres semblent s'inspirer de l'esthétique des estampes japonaises (très à la mode en France dans les années 1910), d'autres encore du style Art nouveau.

29. Je ne reviens pas sur cette caractéristique générale de la démarche cahunienne pour avoir amplement illustré cette conception ailleurs: Andrea Oberhuber, «Cross 


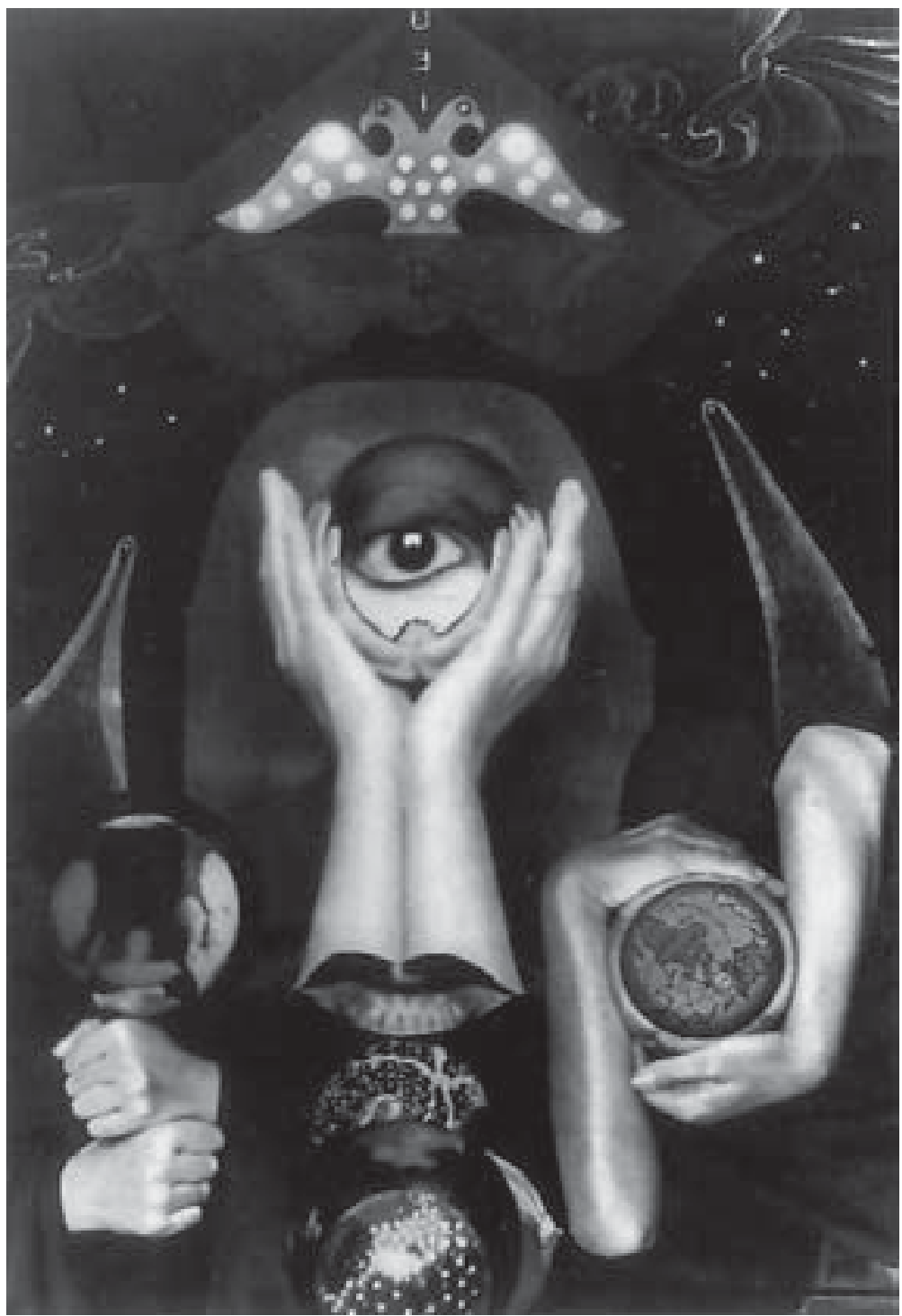

Fig. 4. Claude Cahun, Marcel Moore, Aveux non avenus, Tableau 1, Photomontage original, 1929-1930, coll. National Gallery of Australia, Canberra. 
que Cahun fera sienne tout au long de son cheminement de l'entre-deux : entre les écoles littéraires, les arts et les médias, les noms et les identités. Conçue comme un procédé formel, une forme de différence qui se manifeste dans un jeu de références entre différents «textes », la pratique de Cahun-Moore établira un jeu spéculaire dans lequel les arts et les médias sont mis au service d'une insolite conception de soi et de l'autre. Toujours renouvelées, ces stratégies intermédiales relèvent de la complémentarité, de la redondance ou de la rupture, de la convergence ou de la divergence entre le «je» et son alter ego. Elles s'affirment définitivement dans le cadre de son projet autobiographique au titre paradoxal Aveux non avenus, désigné dès les premières pages comme une «aventure invisible» pour être modifiée un peu plus loin en «aventure sentimentale» (ANA, p. 1 et 3). «Invisible» et «sentimental», tels sont les mots d'ordre qui circonscrivent en effet une entreprise borderline réalisée par Cahun et Moore entre 1919 et 1930, année de publication des Aveux. Les «essais poèmes » ou les «poèmes essais ${ }^{30}$ » permettent le dialogue des genres, dialogue aussi entre le texte et les images, dialogue, finalement, entre l'auteure et l'artiste plasticienne. C'est à travers le mariage d'antinomies et la juxtaposition d'éléments disparates que le texte et les dix photomontages intercalés s'inscrivent dans l'esthétique surréaliste: faire exploser le cadre habituel de l'écriture et laisser arriver le surréel, conjuguer le rêve et la réalité, déclencher une dynamique de l'écart par différents procédés d'écriture. Dans cette quête à la fois d'un ailleurs et d'un autrement, les photomontages viennent troubler les énoncés fragmentaires qui demeurent le plus souvent opaques. Il est évident qu'aux images ne revient aucune fonction «illustrative». Mais alors, quelle autre fonction leur attribuer? Dans une première lecture, elles sont sans aucun doute le gage et l'expression d'une étroite collaboration, d'une complicité exemplaire entre deux créatrices. Et, dans une seconde tentative d'explication, de nature plus sémiotique, ces photomontages revêtent la forme chiffrée d'une déclaration d'amour d'une artiste

gender meets cross media: Claude Cahuns Maskeraden », dans Katharina Hanau et al. (dir.), Geschlechter Differenzen. Beiträge zum 14. Nachwuchs-Kolloquium der Romanistik, Bonn, Romanistischer Verlag, 1999, p. 123-134; "Pour une esthétique de l'entre-deux: à propos des stratégies intermédiales dans l'œuvre de Claude Cahun ", Narratologie, nº 6 , 2004 (à paraître).

30. Le préfacier Pierre Mac-Orlan hésite entre les deux désignations génériques tant ce texte hybride se dérobe à toute classification simple. (ANA, p. III) 


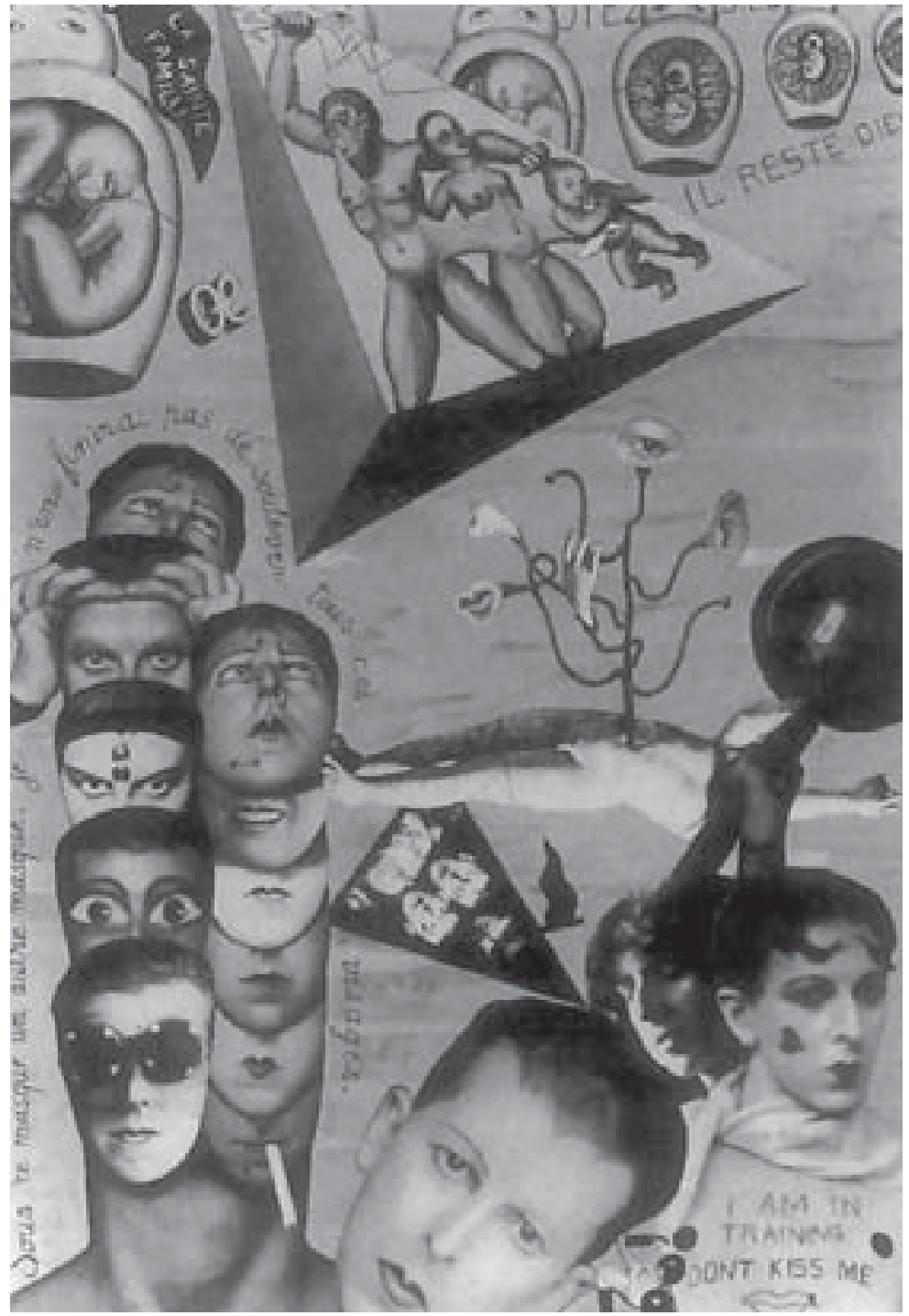

Fig. 5. Claude Cahun, Marcel Moore, Aveux non avenus, Tableau X, Photomontage, 1929-1930, coll. privée (avec l'aimable autorisation de la Galerie Zabriskie, New York). 
à l'autre. Cette déclaration d'amour se traduit par le biais des bouches et des mains, symboles du désir lesbien selon Abigail Solomon-Godeau et Honor Lasalle².

\section{Autre, altérité, autrement}

Les notions de l'autre, de l'altérité et de l'autrement sont le fil conducteur qui garantit la cohérence d'une conception artistique à première vue d'œil inhabituelle et donc déconcertante, disparate parce que protéiforme. L'altérité prend sa source dans le choix de différents pseudonymes (Claude Courlis, Daniel Douglas, Claude Cahun et, pendant la Seconde Guerre mondiale, Der namenlose Soldat; Marcel Moore pour Suzanne Malherbe); elle se prolonge dans la réécriture telle que pratiquée dans Vues et visions et dans Héroïnes, mais également dans l'attribution de nouvelles identités, c'est-à-dire dans ce que Peter Weibel appelle le «libre jeu entre corps, gender et sujet ${ }^{32}$ ». L'être et le paraître sont constamment en concurrence et brouillent les pistes, faisant du sujet unique un être ambigu et ambivalent. Être autrement, s'écrire autrement, se mettre en théâtre dans un éternel jeu de rôles auquel seront convoqués décors, masques et déguisements, telle est la devise de Cahun-Moore. On peut se demander, si «l'aventure invisible» n'est pas celle qui ne laisse pas de traces évidentes, trop facilement lisibles, et qu'au cœur de leur préoccupation se situe l'exploration d'un univers en partie impénétrable. Ainsi seraient-elles à l'origine d'un onirocosme déchiffrable uniquement par elles-mêmes - et peut-être par quelques initiés, leurs amis intimes. Ainsi inventeraient-elles un langage idiosyncrasique dont peut se doter un couple au cours d'une vie commune.

Ce que nous pouvons toutefois comprendre de ce langage, c'est avant tout le principe du double et du multiple, comme si Cahun et Moore voulaient une fois pour toutes renoncer à l'idée de l'Un et de l'unicité, de l'original et de la

31. Abigail Solomon-Godeau, Honor Lasalle, «Surrealist Confession: Claude Cahun's Photomontages», p. 11-13; Abigail Solomon-Godeau, Honor Lasalle, «The Equivocal "I": Claude Cahun as Lesbian Subject», dans Shelley Rice (dir.), Inverted Odysseys, p. 111-125, Abigail Solomon Godeau propose une lecture nuancée de l'impact du lesbianisme sur l'œuvre cahunienne.

32. Peter Weibel parle de «freies Spiel zwischen Körper, Geschlecht und Subjekt » par lequel Cahun préfigure dans ses mises en scène de soi des phénomènes comme le body art des années 1979 et la gender performance de bon nombre d'artistes contemporains. (Peter Weibel, «Alias aliter oder das Subjekt als Sprachspiel : Claude Cahun - Verschieber, Diktatur der Dyade m/w», dans Claude Cahun. Bilder, p. Xxxiv) 


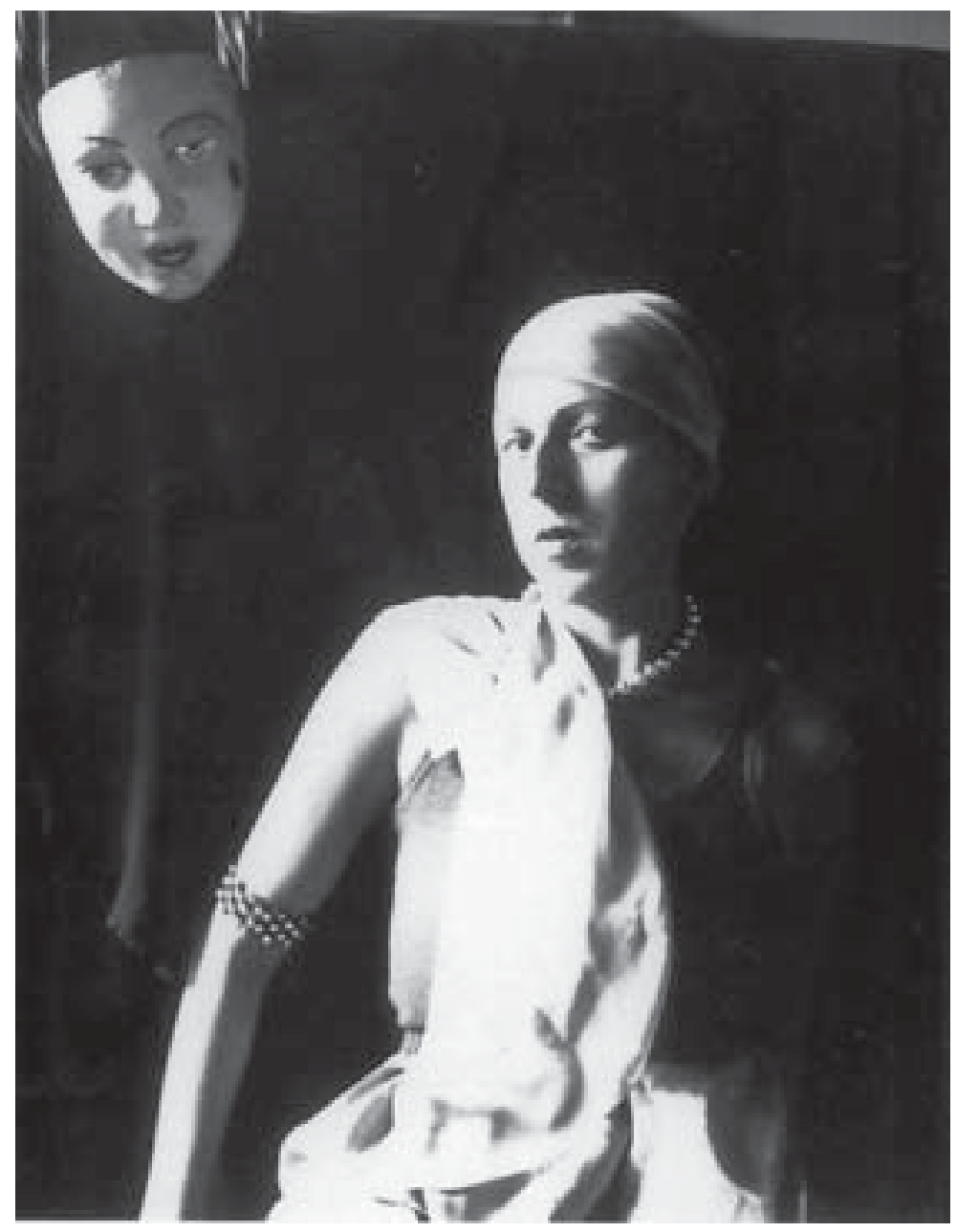

Fig. 6. Claude Cahun, Autoportrait, vers 1928, coll. Musée des Beaux-Arts, Nantes.

copie. Il est vrai que le motif du double est omniprésent dans les autoportraits réalisés entre 1912 et 1947. C'est grâce au jeu des masques que le «je» se dote toujours de nouveaux visages. La célèbre phrase, souvent citée depuis, insérée dans le «Tableau X» (fig. 5), rend ces mascarades explicites: «Sous ce masque un autre masque. Je n'en finirai pas de soulever tous ces visages. (ANA, p. 212) Cependant, à force de trop s'amuser avec les masques, la décalcomanie peut réserver des surprises: 
Devant le miroir un jour d'enthousiasme, on applique trop son masque, il vous mord à la peau. Après la fête, on soulève un coin pour voir... Décalcomanie manquée. On s'aperçoit avec horreur que la chair et le cache sont devenus inséparables. Vite, un peu de salive; on recolle le pansement sur la plaie ${ }^{33}$.

La duplicité atteint son apogée dans les photomontages où le double rencontre d'autres doubles pour finalement se fondre en multiples. C'est du moins l'idée que suggère le «Tableau IX» (ANA, p. 180, fig. 7) : le photomontage présente l'assemblage de la même image de jeunesse de Cahun, les sept autoportraits formant un nœud gordien, et ce, d'autant plus que six des images sont emboîtées l'une dans l'autre (à la manière dont sont attachées des jumelles siamoises) et que des fragments de bras et de jambes y sont entremêlés. Le dédoublement, voire la «spectralisation du “je” participe d'une théâtralisation de l'expression ${ }^{34}$ ", à laquelle est reliée, comme chez la plupart des autobiographes et performance artists, un narcissisme affirmé qui va de pair avec un exhibitionnisme inéluctable ${ }^{35}$.

\section{L'AMOUR AU PRÉCIPICE DU NARCISSISME?}

Face à cette altérité surabondante, le narcissisme que révèlent les textes littéraires, les autoportraits et la plupart des photomontages, apparaît comme l'ultime paradoxe. Mais au fond, tout projet d'autoreprésentation n'est-il pas inévitablement porté par un élan narcissique, comme nous venons de le constater avec Leiris? C'est dans la deuxième partie d'Aveux non avenus, intitulée « Moi-même (faute de mieux)» que Cahun convoque à plusieurs reprises la figure de Narcisse, symbole parfait de notre société occidentale:

33. Claude Cahun, "Carnaval en chambre », La ligne de cour, $n^{\circ} 4$, mars 1926, p. 48-49. On consultera dans ce contexte l'autoportrait datant d'environ 1928 (Claude Cahun. Bilder, p. 27, fig. 6), sur lequel Cahun semble avoir enlevé l'un de ses masques (flottant en haut à gauche) sans encore en connaître le résultat; son regard sceptique dans l'objectif est significatif à cet égard.

34. Martine Antle, «Les femmes photographes du surréalisme», dans Georgiana M. M. Colvile et Katherine Conley (dir.), La femme s'entête: la part du féminin dans le surréalisme, p. 92.

35. Michel Leiris avoue ce penchant dès les premières lignes de L'âge d'homme. Loin de vouloir donner toutefois dans le narcissisme classique, la description de son physique se termine sur sa «laideur humiliante» (Michel Leiris, L'âge d'homme, Paris, Éditions Gallimard, 1939, p. 24). Par conséquent, le narcissisme se voit détourné chez lui vers le dégoût de soi-même. 


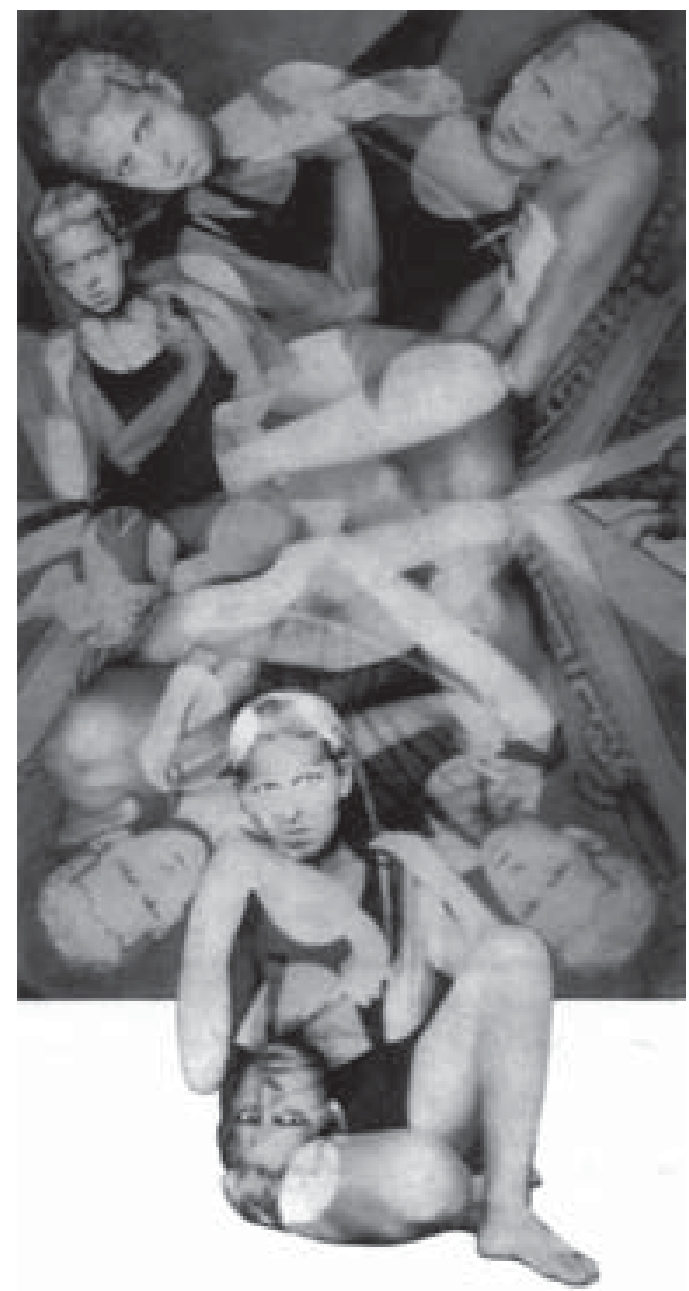

Fig. 7. Claude Cahun, Marcel Moore, Aveux non avenus, Tableau IX, Photomontage, 1929-1930, Éditions du Carrefour, Paris.

Le mythe de Narcisse est partout. Il nous hante. Il a sans cesse inspiré ce qui perfectionne la vie, depuis le jour fatal où fut captée l'onde sans ride. Car l'invention du métal poli est d'une claire étymologie narcissienne. Le bronze - l'argent - le verre: nos miroirs sont presque parfaits. (ANA, p. 38 )

Méfions-nous de toute conclusion simpliste qui verrait derrière ce type d'affirmation générale (dont regorgent les Aveux) le credo d'un «je » narcissique. Car, quelques lignes plus loin, ce même «je» prend ses distances par 
rapport à l'idée de vouloir «fixer l'image dans le temps comme dans l'espace »: " "Miroir", "fixer", voilà des mots qui n’ont rien à faire ici ». (ANA, p. 38) L'évocation réitérée du mythe de Narcisse tout au long de cette deuxième partie est moins l'éloge d'une philautie, d'un «[s]elf-love ${ }^{36}$ », qu'une invitation à réfléchir sur le regard: regard - sans fard - sur soi-même, et regard porté sur l'autre. Cette lecture se voit confirmée par l'analyse du photomontage ouvrant la partie «Moi-même». Dominé par un immense œil qui, semblable à une planète lunaire, occupe la partie inférieure de l'image, le «Tableau III» (ANA, p. 26, fig. 8) thématise explicitement le regard: par l'œil, évidemment, mais aussi par le biais d'un miroir de femme tenu par une main, dans lequel est reflétée la partie supérieure seulement du visage de Cahun, soit ses yeux et son front. À bien y regarder, on s'aperçoit que l'œil-planète est l'agrandissement surhumain de l'œil droit de Cahun. Reste à savoir à qui s'adresse ce double regard, à qui s'adressent tous ces regards directs dans l'objectif de l'appareil photo, autre type de miroir. Aux spectateurs que nous sommes, certes, et nous sommes plus d'une fois déconcertés par ce regard fixe et percutant. Mais il s'adresse sans doute aussi à celle qui, derrière la caméra, dirige savamment la mise en scène du sujet et appuiera, le moment venu, sur le déclencheur, afin de fixer cette image parmi tant d'autres. On l'aura compris: l'œil et le regard ont une fonction métaphorique dans cette démarche qui se veut aussi bien esthétique que psychologique. Si, de plus, il faut voir derrière ces nombreux autoportraits, à défaut d'une existence certaine, le besoin de s'assurer d'une existence momentanée au sens de «Je me vois, donc je suis", le regard de Cahun prolonge la scène vers le hors-champ, là où l'on devine son alter ego. Par-delà l'étonnante fixité du regard comme expression d'une souveraineté, ce regard off établit, en définitive, le lien avec l'Autre. En même temps, il s'inscrit contre la pratique photographique dominante, celle de Man Ray, de Brassaï, d’André Kertesz ou de Raoul Ubac, par exemple, qui, la plupart du temps, montrent des corps de femmes

36. L'elliptique «Self-love» joue à deux reprises le rôle d'intertitre ouvrant les passages suivants: «La mort de Narcisse m’a toujours paru la plus incompréhensible. Une seule explication s'impose : Narcisse ne s'aimait pas. Il s'est laissé tromper par une image. Il n'a pas su traverser les apparences » (ANA, p. 36) et «Une main crispée sur un miroir - une bouche, des narines palpitantes - entre des paupières pâmées, la fixité folle de prunelles élargies... Dans l'horizon brutale d'une lampe électrique, en blond, mauve et vert sous les étoiles, voilà tout, par pudeur! ce que je voudrais éclairer du mystère : le néo-narcissisme d'une humanité pratique.» (ANA, p. 37) 


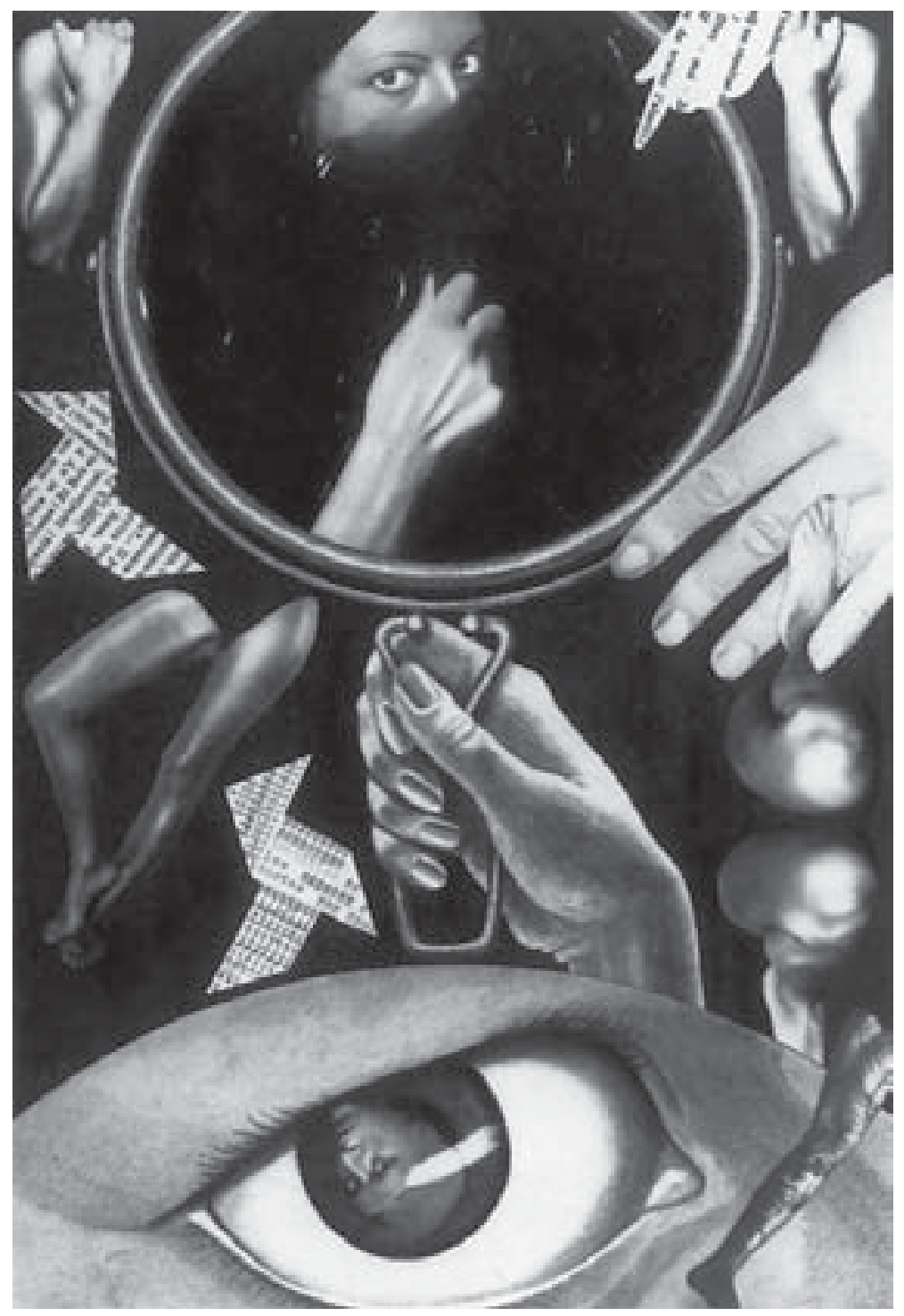

Fig. 8. Claude Cahun, Marcel Moore, Aveux non avenus, Tableau III, Photomontage, 1929-1930, coll. privée (avec l'aimable autorisation de la Galerie Zabriskie, New York). 
fragmentés, distordus et, surtout, des visages aux yeux fermés. Ce qui, de prime abord, paraît donc profondément narcissique, peut se révéler, comme dans un bain chimique, une double, sinon une triple remise en question de cette impression de départ: d'abord, parce que l'autoportrait, comme forme traditionnelle d'autoreprésentation d'un individu à un moment précis de sa vie, trouve son unicité démentie à travers la démultiplication ad infinitum; ensuite, parce que le sujet récuse le jeu de la femme-objet devant l'objectif telle qu'aimait la glorifier la photographie et la peinture surréalistes; et enfin, parce que, tout comme dans l'écriture autobiographique, le «je» appelle constamment un «tu». Chez Cahun, le narcissisme ouvre donc un espace de transition ${ }^{37}$ où, comme dans un perpetuum mobile l'un renvoie toujours à l'autre sans jamais atteindre un état stable, où sont accueillis non seulement différents regards et identités (remodelées par des postures et des déguisements, des masques et des mascarades qui puisent dans le fonds quasiment illimité d'un imaginaire débridé), mais également d'autres voix, des spectres et des figures transfuges. Ce besoin d'autres présences entraîne le récit des Aveux dans une dimension dialogique; c'est comme si l'introspection nécessitait un autre, le double, l'alter ego tant recherché, afin de pouvoir établir un dialogue avec lui et, au bout du compte, de s'avouer. La voix d'autrui est à l'écriture du moi ce qu'est le miroir à l'autoportrait photographique: se refléter dans une surface lisse, moins par inclination narcissique, bien que ce penchant ne soit négligeable, que pour faire parader devant le lecteur-spectateur les multiples voix et visions intérieures d'un univers onirique.

Rattachés l'un à l'autre par un anneau imperceptible à l'œil nu, le(s) texte(s) d'Aveux non avenus et les photomontages peuvent se lire comme des trompel'œil. Ostensiblement, il leur manque une dimension, celle qui nous intrigue et nous interpelle parce que cette dimension en moins ouvre une brèche à la séduction, comme le constate Baudrillard à propos du miroir et du narcissisme : «La séduction est ce dont il n’y a pas de représentation possible, parce que la distance entre le réel et son double, la distorsion entre le Même et l'Autre y est abolie $^{38}$.» En effet, Cahun ne cherche ni en soi ni chez Moore l'attraction du

37. Martine Antle parle d'un «espace de récréation» pour désigner la dimension ironique qui sous-tend bon nombre des autoportraits de femmes surréalistes. (Martin Antle, «Les femmes photographes du surréalisme», p. 93)

38. Jean Baudrillard, De la séduction, Paris, Éditions Galilée, coll. «L'espace critique », 1979, p. 93 
même ou l'exaltation mimétique de sa propre image. Bien au contraire, consciente des effets trompeurs du mirage de la ressemblance, plutôt que de vouloir retrouver le reflet identique de soi-même sur une surface miroitante, elle est obsédée par l'idée de la métamorphose. Vers la fin de son voyage introspectif, le «je» ne termine-t-il pas ses autoréflexions sur un souhait lucide: «Vive et grandisse en moi celui, celle - ou simplement ce - qui me permit, jeune encore, de comprendre que je ne puis, toucher, transformer, que moi-même » (ANA, p. 233)? Se transformer constamment deviendrait alors la réponse à un univers «en humeur de métamorphose» (ANA, p. 233). L’idée de se métamorphoser, de «changer de peau: arrache-moi la vieille» (ANA, p. 232) - tel est l'impératif mis en exergue à la dernière partie des Aveux - traduit toujours ce même désir de tendre le miroir à ceux qui lisent le texte, regardent les photomontages. Si dans le cas des écrits et des images est déclenché un sentiment vertigineux devant un gouffre qui s'ouvre, c'est probablement parce que le spectacteur aperçoit dans ce miroir tendu vers lui une absence de profondeur révélant un «abîme superficiel qui n’est pas séduisant et vertigineux pour les autres que parce que chacun est le premier à s'y abîmer ${ }^{39}$ ». Ainsi, la séduction porte en elle autant d'(at)traits de narcissisme que d'altruisme. Car, l'ultime destinataire de ces œuvres d'art n'est-elle pas l'âme-sœur de Cahun, Marcel Moore? Celle qui est toujours à ses côtés, qui l'inspire et, du début jusqu'à la fin, apporte son concours à une grande partie de l'œuvre littéraire et photographique? N'est-ce pas elle en premier qu'il s'agit de séduire? Je suggère, comme grille de lecture de la démarche de Cahun-Moore, une stratégie de la séduction mutuelle qui, toujours selon Baudrillard, s'apparente à celle du leurre en se présentant comme un miroir de l'inconscient et du désir : «Séduire, c'est mourir comme réalité et se produire comme leurre ${ }^{40}$. "C'est dans la répétition que l'illusion du leurre est capable de conférer une réalité absolue à l'objet-sujet qui réussit à capter le faucon. Le leurre confirme, tout compte fait, la reconnaissance du pouvoir incontesté du rituel de la séduction. Comme sur une scène de théâtre, Cahun répète différents rôles, essaie des costumes et des masques, se complaît dans des postures tantôt mythologiques, tantôt ironiques ou parodiques. Ces diverses métamorphoses fixées sur négatif, puis tirées dans le but d'en faire un usage personnel ${ }^{41}$, ne sont donc pas a priori destinées à séduire

39. Jean Baudrillard, De la séduction, p. 95.

40. Jean Baudrillard, De la séduction, p. 96.

41. Voir à ce propos les remarques critiques de Catherine Gonnard dans son article 
quiconque, mais principalement la «protagoniste » elle-même et sa «metteure en scène", Moore. Dans un deuxième temps, ces images semblent avoir été montrées ou offertes à certains bons amis du couple. Le caractère privé des photographies, surtout des autoportraits - à quelques exceptions près ${ }^{42}-$, contrairement à la publication d'une majeure partie des œuvres littéraires de Cahun, soulève la question du lien qui existe entre la production artistique du couple Cahun-Moore et la sphère publique. Le choix du petit format de la plupart des tirages et le fait qu'ils ne sont pas signés confirment l'hypothèse du but privé auquel étaient apparemment destinées ces photographies. Elles acquièrent pourtant un statut public à travers leur recyclage dans les dix photomontages insérés dans Aveux non avenus. Faut-il en conclure que les autoportraits constituaient avant tout un fonds d'images qui devaient, à leur tour, nourrir les photomontages puisqu'on y retrouve, en fragments, un grand nombre de ces fascinants autoportraits? Ou alors, Cahun-Moore faisaient-elle la part des choses entre les mises en scène de soi à caractère strictement privé et la conception de portraits d'artistes, de photomontages et d'objets surréalistes voués dès le départ à la publication ou à l'exposition? Tant qu'on ne dispose d'autres sources nous

sur la première exposition des autoportraits de Cahun en France, au Musée d'art moderne de la ville de Paris: Catherine Gonnard, "Claude Cahun photographe. Un paradoxe lesbien ", Lesbia magazine, $\mathrm{n}^{\circ}$ 141, septembre 1995, p. 30-32. La journaliste insiste sur le caractère «obscène » des agrandissements des photographies qui, sans indiquer leur format originel, tromperaient le spectateur dans sa réception et ne rendraient pas compte de l'intimité liée aux autoportraits. Elle y souligne également le paradoxe entre présentation publique et destination privée des photographies, de même que celui qui consiste à placer les œuvres de Cahun dans le seul contexte de la modernité sans mention aucune de son homosexualité, point d'ancrage de leur production. C'est sur ce dernier point qu'insiste également Laura Cottingham, Cherchez Claude Cahun, p. 19-22.

42. L’un des rares autoportraits signés, "Que me veux-tu? (fig. 9), parut en 1929 sur la page de couverture du recueil Frontières humaines de Ribemont-Dessaignes avant d'être reconfiguré dans le «Tableau IV» d'Aveux non avenus. En 1930, Cahun publia dans le numéro 5 de Bifur, modeste revue parisienne, l'«Autoportrait» anamorphotique (environ 1929), c'est-à-dire une variante de l'autoportrait datant de 1928 (voir Claude Cahun. Bilder, p. 42 et 43), adhérant ainsi ouvertement à l'esthétique surréaliste. C'est en 1936 seulement que Cahun rendit vraiment public son travail en participant avec «Souris valseuses» et «Un air de famille» à la grande Exposition surréaliste d'objets organisée à la Galerie Charles Ratton à Paris. Un an plus tard, à la publication du Coeur de pic de Lise Deharme, le public prit à nouveau connaissance des œuvres de Cahun : le livre pour enfants est «illustré de vingt photographies de Claude Cahun». 


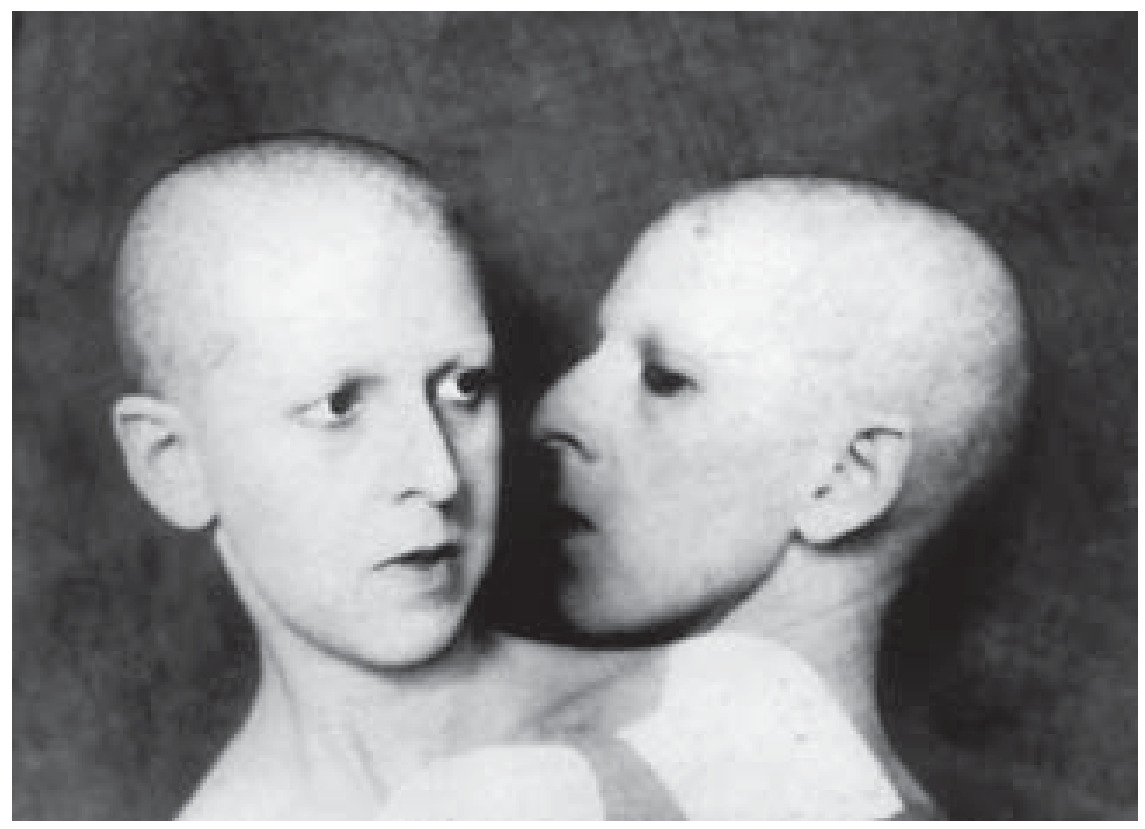

Fig. 9. Claude Cahun, Que me veux-tu ?, 1928, coll. privée, Paris.

informant sur la relation "privée-publique ${ }^{43}$ » qu'entretenait le couple avec ses photographies, la question demeure entière. Mais elle nous amène sur un autre terrain, celui de l'opinion publique face au désir lesbien.

\section{LE DÉSIR LESBIEN, UN « FAUX MASQUE »?}

La circulation interne des images de Cahun et de Moore en dit long également sur les interdits et les tabous auxquels se heurtaient les amantes dans l'espace public. C'est en ce sens que les travaux du couple revêtent une fonction émancipatoire: les multiples figures du «je» nient l'idée d'une identité comme la seule «vraie» et, par là, celle de l'idée d'une fausse identité car «déviante».

43. Selon François Leperlier, l'artiste entretenait une relation «privée» avec ses photographies. Il donne comme explication le refus de vouloir faire carrière en photographie en faveur d'un dilettantisme revendiqué et d'un penchant pour «l'indéfini»: François Leperlier, "Claude Cahun », dans Mise en scène, catalogue d'exposition, Londres, Institute for Contemporary Arts, 1994, p. 16-20. 
Ceci est d'autant plus important à une époque qui stigmatise le désir lesbien comme «faux masque ${ }^{44}$ ", ou dans une culture qui considère, comme l'avait analysé Joan Rivière, la féminité comme mascarade («womanliness as a mas$\left.q_{\text {querade }}^{45} »\right)$. Nombreux sont ceux qui, dans l'entre-deux-guerres, voient dans les couples lesbiens une déviation de l'émancipation des femmes; le scandale provoqué par La garçonne de Victor Margueritte confirme cette stigmatisation. Le discours médical et psychiatrisant du début du $\mathrm{XX}^{\mathrm{e}}$ siècle sur le phénomène du «travestissement» en général et des «femmes travesties» en particulier contribue largement à la pathologisation d'une pratique érotique autre. Ce qui est vécu comme une libération sociale et sexuelle est interprété, selon le modèle médical hétérosexuel, comme une pratique immorale, malsaine, bref contrenature $^{46}$. Jusqu'à quel point faut-il voir dans l'homosexualité féminine une réaction à l'oppression sociale - dans le dessein de contourner des barrières professionnelles - , un désir de s'affranchir des limites hommes/femmes et des contraintes sociopolitiques véhiculées par cette dichotomie? La réponse n’est pas généralisable tellement le phénomène du travestissement, qui n'est que l'apparence d'un bouleversement plus profond des rôles sexués, revêt dans chaque cas ou chaque couple une motivation bien particulière. Mais, ce que les phénomènes

44. Voir à ce propos Laurie Monahan, «Claude Cahuns radikale Transformationen », Texte zur Kunst, $\mathrm{n}^{\circ}$ 11, septembre 1993, p. 103; Elisabeth Lebovici, "I am in training. Don’t kiss me” ", Claude Cahun photographe, p. 21 ; Laura Cottingham, «Betrachtungen zu Claude Cahun ", p. XvII.

45. Tel est le titre d'un article publié pour la première fois en 1929 dans le Journal of Psychoanalysis (repris dans Joan Rivière, The Inner World and Joan Rivière. Collected Papers 1920-1958, London, Karnac Books, 1991, p. 90-101). Dans cet article, la psychanalyste rendait compte d'une certaine attitude de (sur-)féminité affichée par des patientes qui voulaient réussir dans le monde des hommes sans provoquer leur méfiance, d'une part, ou pour qui la féminité était le moyen de se soumettre aux jeux de rôles traditionnels afin d'éviter la concurrence masculine, d'autre part. Dans les deux cas, Rivière compare la «féminité » à une mascarade, autrement dit à une stratégie typiquement féminine censée garantir, du moins en apparence, l'ordre social par le jeu sexué traditionnel, ce qui équivaut, selon l'analyste, à la négation même d'une féminité «essentielle».

46. Voir Marie-Hélène Bourcier, «Des "femmes travesties" aux pratiques transgenres: repenser et queeriser le travestissement », CLIO, Histoire, femmes et sociétés, $n^{\circ}$ 10, 1999, p. 118-121. L'auteure souligne le prolongement de cette vision depuis le XVIII ${ }^{\mathrm{e}}$ siècle, pour lequel le travestissement était une «perversion contre-nature » qui procurait des «orgasmes contre-nature»; Freud, à son tour, concevra le fétichisme comme une perversion uniquement masculine. 


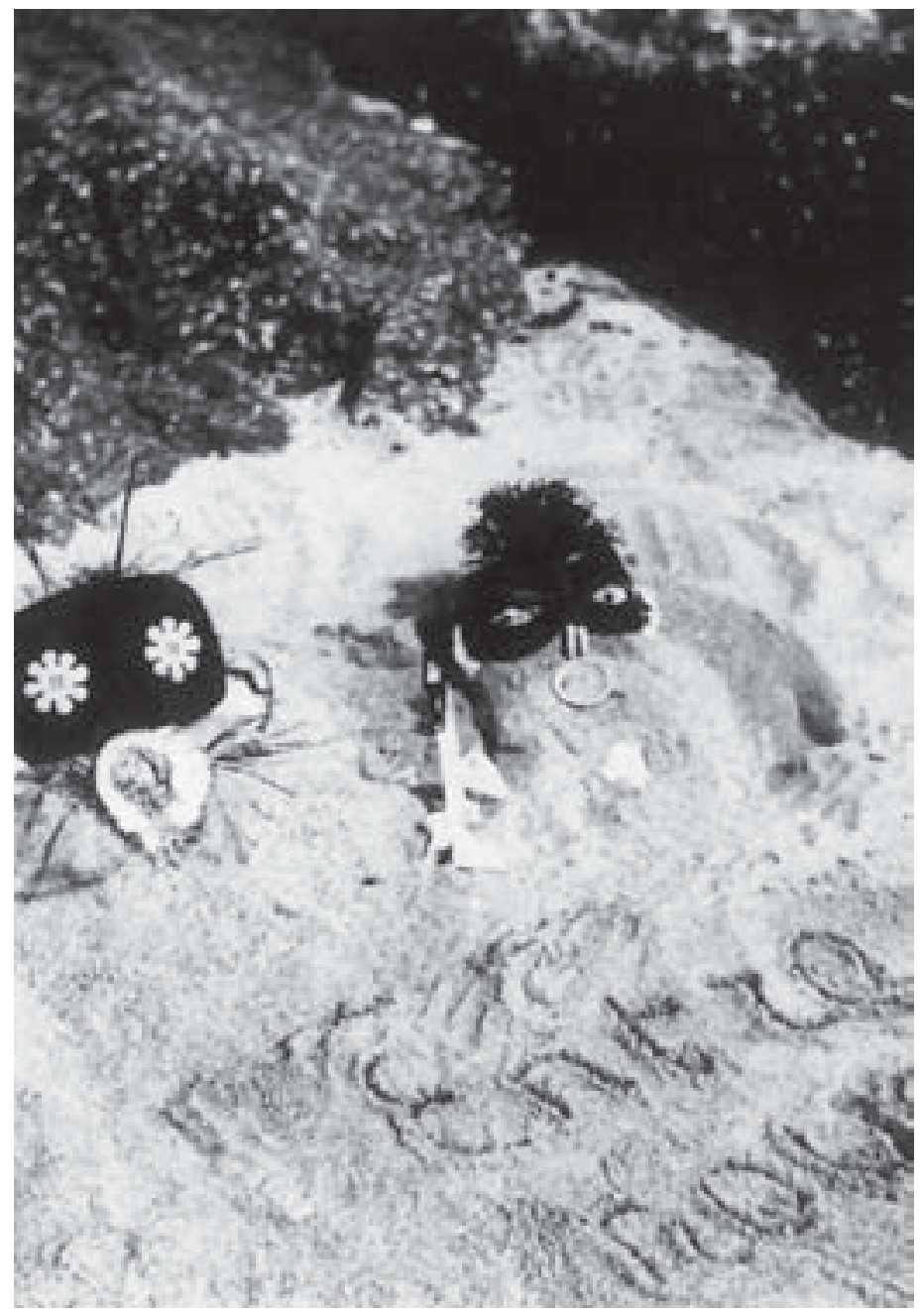

Fig. 10. Claude Cahun, Entre nous, 1926, Galerie Berggruen.

historiques de la «nouvelle femme» et du désir lesbien soulevaient comme question fondamentale commune, c'était la nécessité de repenser la norme hétérosexuelle sur les plans philosophique, médical, social et politique, de comprendre cette nouvelle apparence et la pratique érotique qui lui est associée comme la déstabilisation des frontières assignées entre les deux sexes, de les accepter comme la crise des catégories perçues volontiers comme stables, de 
briser, d'après Judith Halberstam, avec la rhétorique binaire du trans- et du cross- pour ne pas reconduire le bipolarisme ${ }^{47}$.

Cahun et Moore, selon le choix des médias et du projet, faisaient leur des idées et des positions souvent à l'opposé des normes sociales et esthétiques, se situant presque systématiquement au-delà de la «normalité». Ces positions, Cahun en particulier les défendait d'autant plus décidément qu'en tant que juive, femme et lesbienne, elle se retrouvait d'office à la périphérie et que non seulement son éthique, mais aussi sa démarche d'artiste devaient forcément différer du centre, se retrouver à l'écart du mainstream. Enracinées dans un éclectisme avoué, les choix esthétiques du couple Cahun-Moore les ont toujours placées dans une atopie radicale qu'elles semblaient rechercher sans cesse ${ }^{48}$. Dans Gender Trouble, Judith Butler se demande dans quelle mesure «identité » équivaut plus à un idéal normatif qu'à un signe descriptif d'expérience. Elle souligne que la «cohérence » et la «continuité » d'une «personne » ne sont pas des signes logiques et analytiques de sa personnalité, mais renvoient à des conventions sociales et à un ordre moral par lesquels les êtres humains sont non seulement définis, mais également normés ${ }^{49}$. Au lieu de se laisser enfermer dans ces conventions, Claude Cahun et Marcel Moore inversent les pôles de cellesci pour imaginer un mode de création, de vie, d'identité et d'amour radicalement différent. Dans leur salon, à l'écart de l'avant-garde surréaliste, elles créent un espace où devient possible leur love story de l'entre-deux: entre deux époques, entre arts et médias, entre deux femmes artistes (fig. 10).

47. Marie-Hélène Bourcier, "Des "femmes travesties" aux pratiques transgenres", p. 128-132. Florence Tamagne propose un portrait général, littéraire et artistique de cette modernité rebelle: Florence Tamagne, Mauvais genre? Une histoire des représentations de l'homosexualité, p. 133-155.

48. Voir Elisabeth Bronfen, «Die Vorführung der Hysterie», dans Aleida Assmann et Heidrun Friese (dir.), Identitäten. Erinnerung, Geschichte, Identität 3, Frankfurt am Main, Suhrkamp Verlag, 1998, p. 256. Bronfen utilise le terme «radikale Atopie» pour désigner, comme l'une des caractéristiques générales de la démarche cahunienne, la transgression des frontières conventionnelles à la recherche permanente d'un Sein, d'une identité indéfinis.

49. Judith Butler, Gender Trouble: Feminism and the Subversion of Identity, New York, London, Routledge Press, 1999 [1990], p. 17. 